Table 1. Prevalence of cognitive impairment in patients with minor/nonNPSLE, inflammatory NPSLE and ischemic NPSLE

\begin{tabular}{lcccc}
\hline & $\begin{array}{c}\text { Global cognitive } \\
\text { functioning }\end{array}$ & $\begin{array}{c}\text { Learning } \\
\text { memory }\end{array}$ & $\begin{array}{c}\text { Executive functioning \& } \\
\text { complex attention }\end{array}$ & $\begin{array}{c}\text { Psychomotor } \\
\text { speed }\end{array}$ \\
\hline All patients & $8(5)$ & $70(46)$ & $57(39)$ & $69(46)$ \\
$\begin{array}{l}(n=151) \\
\text { Inflammatory } \\
\text { NPSLE }\end{array}$ & $3(13)$ & $14(58)$ & $12(50)$ & $12(50)$ \\
$\begin{array}{l}\text { (n=24) } \\
\text { Ischemic NPSLE }\end{array}$ & $0(0)$ & $6(50)$ & $2(17)$ & $2(17)$ \\
$\begin{array}{l}(n=12) \\
\text { Minor/non-NPSLE }\end{array}$ & $5(4)$ & $50(44)$ & $43(38)$ & $55(49)$ \\
(n=115) & & & & \\
\hline
\end{tabular}

NPSLE = neuropsychiatric systemic lupus erythematosus.Data represent $\mathrm{n}(\%)$ of patients with cognitive impairment in the mentioned cognitive domain. Cognitive impairment was defined as cognitive function at least 1SD lower than the mean of an age, sex and education matched general Dutch population. The percentages were calculated from total number of patients with available scores: Global functioning: 23/24 inflammatory NPSLE and 113/115 minor/non-NPSLE; psychomotor speed 113/115 minor/non-NPSLE, executive function \& complex attention: also 113/115. All tests were available for ischemic NPSLE.

Disclosure of Interests: None declared

DOI: 10.1136/annrheumdis-2021-eular.881

\section{POS0715 $\quad$ QUANTIFYING THE PSYCHOSOCIAL IMPACT OF QUANTIFYING THE PSYCHOSOCIAL IMPACT
UNDIFFERENTIATED CONNECTIVE TISSUE DISEASE (UCTD)}

C. Siegel ${ }^{1,2}$, E. Sevim ${ }^{3,4}$, B. Stamm ${ }^{5}$, J. Vega ${ }^{5}$, J. Kleinman ${ }^{6}$, J. Barnhill ${ }^{7}$, M. Lockshin ${ }^{2,5}$, L. Sammaritano ${ }^{2,5}$, M. Barbhaiya ${ }^{2,5} .{ }^{1}$ Hospital for Special Surgery, Rheumatology, New York, United States of America; ${ }^{2}$ Weill Cornell Medicine, Internal Medicine, New York, United States of America; ${ }^{3}$ Montefiore Medical Center, Internal Medicine, Bronx, United States of America; ${ }^{4}$ Albert Einstein College of Medicine, Internal Medicine, Bronx, United States of America; ${ }^{5}$ Hospital for Special Surgery, Barbara Volcker Center for Women and Rheumatic Diseases, New York, United States of America; ${ }^{6}$ Hospital for Special Surgery, Department of Social Work Programs, Division of Rheumatology, New York, United States of America; ${ }^{7}$ Weill Cornell Medicine, Psychiatry, New York, United States of America

Background: Only half of patients diagnosed with SLE fulfill classification criteria; the rest have "SLE-like" illnesses such as UCTD. SLE patients are known to experience impaired health-related quality of life (HRQoL) and significant anxiety, depression, and fatigue, ${ }^{1}$ yet the psychosocial aspects of UCTD are less established. In a qualitative study, we found that most UCTD patients had engaged in psychotherapy and felt additional support was needed. ${ }^{2}$

Objectives: Using multiple validated instruments, this study aims to quantify the psychosocial impact of UCTD.

Methods: The Hospital for Special Surgery UCTD and Overlap Registry includes UCTD patients aged $\geq 18$ years with ANA $\geq 1: 80$ and $\geq 1$ sign or symptom of rheumatic disease who do not fulfill classification criteria for a defined CTD. We administered the 36-Item Short Form Health Survey (SF36), General Anxiety Disorder-7 (GAD-7), Beck Depression Inventory (BDI), and Fatigue Severity Scale (FSS) to all patients to assess HRQoL, anxiety, depression, and fatigue. Instruments were scored based on established algorithms and results were summarized using predefined scales and severity thresholds.

Results: The composite questionnaire was administered to 85 UCTD patients and completed by $75(97.3 \%$ female, $60 \%$ white, mean age \pm SD $48.8 \pm 13.6$ years). The SF-36 Physical Component Summary mean score was 37.8 and Mental Component Summary mean score was 41.1. Across the 8 SF-36 subscales, mean scores were lowest for role limitations due to physical health (39.3) and vitality (39.7) and highest for physical functioning (67.2), role limitations due to emotional health (67.1), and mental health (67.1). Approximately half of UCTD patients reported anxiety (GAD-7 $\geq 6$ ); $20 \%$ had moderate/severe anxiety (GAD-7 $\geq 10$ ). The prevalence of depression $(\mathrm{BDI} \geq 14$ ) was $26.7 \% ; 13.3 \%$ had moderate/severe depression (BDI $\geq$ $20)$. Fatigue (FSS $\geq 3$ ) was reported by $82.8 \%$ of patients (median FSS score of 4.7) [Table 1].
Table 1. Psychosocial Survey Scores of Patients with Undifferentiated Connective Tissue Disease $(n=75)$

\begin{tabular}{|c|c|c|}
\hline \multirow{3}{*}{$\begin{array}{l}\text { 36-Item Short Form Health Survey } \\
\text { (SF-36) }\end{array}$} & Physical Component Summary & $38.2(11.2)$ \\
\hline & Physical functioning & $67.2(26.3)$ \\
\hline & •Role-Physical & $39.3(46.3)$ \\
\hline \multirow[t]{7}{*}{ Range $1-100-$ Mean $(S D)^{*}$} & •Bodily Pain & $49.5(22.1)$ \\
\hline & oGeneral Health & $42.9(21.5)$ \\
\hline & Mental Component Summary & $41.3(10.7)$ \\
\hline & -Vitality & $39.7(21.7)$ \\
\hline & -Social Functioning & $59.3(25.9)$ \\
\hline & 'Role-Emotional & $67.1(41.9)$ \\
\hline & oMental Health & $67.1(18.3)$ \\
\hline \multirow{3}{*}{$\begin{array}{l}\text { Generalized Anxiety Disorder-7 } \\
\text { (GAD-7) }\end{array}$} & None $[0-5]$ & $38(50.7)$ \\
\hline & Mild [6-10] & $22(29.3)$ \\
\hline & Moderate [11-15] & $14(18.7)$ \\
\hline Range $0-21-N(\%)^{\star *}$ & Severe [16-21] & $1(1.3)$ \\
\hline \multirow[t]{2}{*}{ Beck Depression Inventory (BDI) } & Minimal [0-13] & 55 (73.3) \\
\hline & Mild [14-19] & $10(13.3)$ \\
\hline \multirow[t]{2}{*}{ Range $0-63-N(\%)^{\star *}$} & Moderate [20-28] & $7(9.3)$ \\
\hline & Severe [29-63] & $3(4.0)$ \\
\hline \multicolumn{2}{|c|}{ Fatigue Severity Scale (FSS) Range 1-7 - Median (IQR) ${ }^{\star *}$} & $4.7(1.5)$ \\
\hline
\end{tabular}

${ }^{*}$ Higher number indicates better health state. ${ }^{* *}$ Higher number indicates greater severity.

Conclusion: UCTD patients have significantly impaired HRQoL and a high prevalence of anxiety, depression, and fatigue, suggesting substantial psychosocial impact of UCTD comparable to that reported in SLE. ${ }^{3,4}$ Impaired HRQoL in UCTD is driven to similar degrees by aspects of physical and mental health. In future studies, we will compare age- and sex- matched UCTD to SLE patients and longitudinally evaluate psychosocial metrics alongside clinical trajectories. REFERENCES:

[1] Dietz B, Katz P, Dall'Era M, et al. Major depression and adverse patient-reported outcomes in systemic lupus erythematosus: Results from a prospective longitudinal cohort. Arthritis Care Res. 2021;73(1):48-54.

[2] Siegel $\mathrm{CH}$, Kleinman J, Barbhaiya $\mathrm{M}$, et al. The psychosocial impact of undifferentiated connective tissue disease on patient health and well-being: A qualitative study. J Clin Rheumatol. In press.

[3] Gu M, Cheng Q, Wang X, et al. The impact of SLE on health-related quality of life assessed with SF-36: A systemic review and meta-analysis. Lupus. 2019;28(3):371-382.

[4] Zhang L, Fu T, Yin R, Zhang Q, Shen B. Prevalence of depression and anxiety in systemic lupus erythematosus: A systematic review and meta-analysis. BMC Psychiatry. 2017;17(1).

Acknowledgements: This project was supported by the Barbara Volcker Center for Women and Rheumatic Diseases and the Robin J. Sillau Memorial Research Fund for Connective Tissue Disease. Dr. Barbhaiya is supported by the Rheumatology Research Foundation Investigator Award.

Disclosure of Interests: None declared

DOI: 10.1136/annrheumdis-2021-eular.929

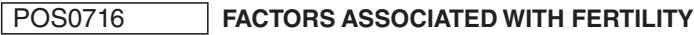 OUTCOMES IN WOMEN WITH SYSTEMIC LUPUS ERYTHEMATOSUS: A SYSTEMATIC REVIEW}

S. Giambalvo ${ }^{1}$, C. Garaffoni ${ }^{1}$, E. Silvagni ${ }^{1}$, F. Furini ${ }^{2}$, M. Govoni ${ }^{1}$, A. Bortoluzzi ${ }^{1}$. ${ }^{1}$ Unit of Rheumatology, Azienda Ospedaliera-Universitaria Sant'Anna, Department of Medical Sciences, University of Ferrara, Ferrara, Italy; ${ }^{2}$ Unit of Rheumatology, Ospedale Maggiore, Bologna, Italy

Background: Fertility is thought to be not reduced in women affected by systemic lupus erythematosus (SLE), however disease-related factors, psychosocial effects of chronic disease as well as medication exposure might impair gonadal function.

Objectives: The aim of this systematic review was to explore clinical, hormonal, serological, instrumental and management factors associated with fertility outcomes in women of childbearing age with SLE.

Methods: This systematic review was conducted following the Preferred Reporting Items for systematic reviews and Meta-analysis (PRISMA) statement. All articles available in English, published from 1972 to 15th August 2020 in Pubmed, EMBASE, Scopus and Cochrane Library. Study selection and data collection were performed by two independent reviewers. All data were extracted using a standardized template. Risk of bias of the included studies was assessed by using the NIH risk-of-bias tool [1] 
Results: Of 788 abstracts, we included in the review 45 studies of which 1 systematic literature reviews, 16 cross-sectional studies, 15 cohort studies, 12 observational studies and 1 case-series study, with a total of 4656 patients. The mean age was $33.5 \pm 5.4$ years, while the mean disease duration was $97.4 \pm 65.2$ months. Figure 1 illustrates the quality of the included studies. Definitions of fertility/premature ovarian failure (POF) adopted in the studies varied in terms of the number of months of amenorrhea considered. Most studies did not use a hormonally based definition of fertility. Clinical factors associated with the development of POF were older age at the time initiation of therapy and older age at the onset of SLE disease. Cyclophosphamide exposure (CYC) and its cumulative dose influenced gonadal function in SLE women, leading to amenorrhoea and ovarian failure, as reported in 19 studies. Mycophenolate, azathioprine, calcineurin inhibitors and steroids seem to be associated with a lower risk of ovarian failure compared to CYC. 3 studies demonstrated that POF was more frequent in patients treated with CYC not receiving gonadotropin-releasing hormone analogues $(\mathrm{GnRH})$ in comparison to those co-treated with $\mathrm{GnRH}$. 11 studies evaluated the impact of damage and disease activity on ovarian reserve in patients with SLE with conflicting evidence. Finally, 18 studies investigated exposure to hormonal and serological factors able to influence fertility outcomes; among others nor Anti-Müllerian Hormone, neither anti-corpus luteum antibodies were associated with POF.

Conclusion: The role of disease activity on fertility in SLE patients is contradictory. Regarding management factors associated with fertility in SLE women of childbearing age, the strongest evidence is about the treatment with CYC and its cumulative dose. Hormonal and serological factors did not impact on fertility outcome but might be used as a surrogate of fertility, especially after treatment with disease-specific drugs. REFERENCES:

[1] Study Quality Assessment Tools NIH. https://www.nhlbi.nih.gov/ health-topics/study-quality-assessment-tools.

[2] Andreoli L. et al. EULAR recommendations for women's health and the management of family planning, assisted reproduction, pregnancy and menopause in patients with systemic lupus erythematosus and/or antiphospholipid syndrome. Ann Rheum Dis. 2017; 76: 476-485

Figure 1 - Risk of bias graph: review authors' judgements about each risk of bias using NIH risk-of-bias tool. 1A: systematic literature review/meta-analysis; 1B: observational studies; $1 \mathrm{C}$ : case series; green box = "yes/low risk of bias"; yellow box = "not applicable/not reported"; red box = "no/potential risk of bias"
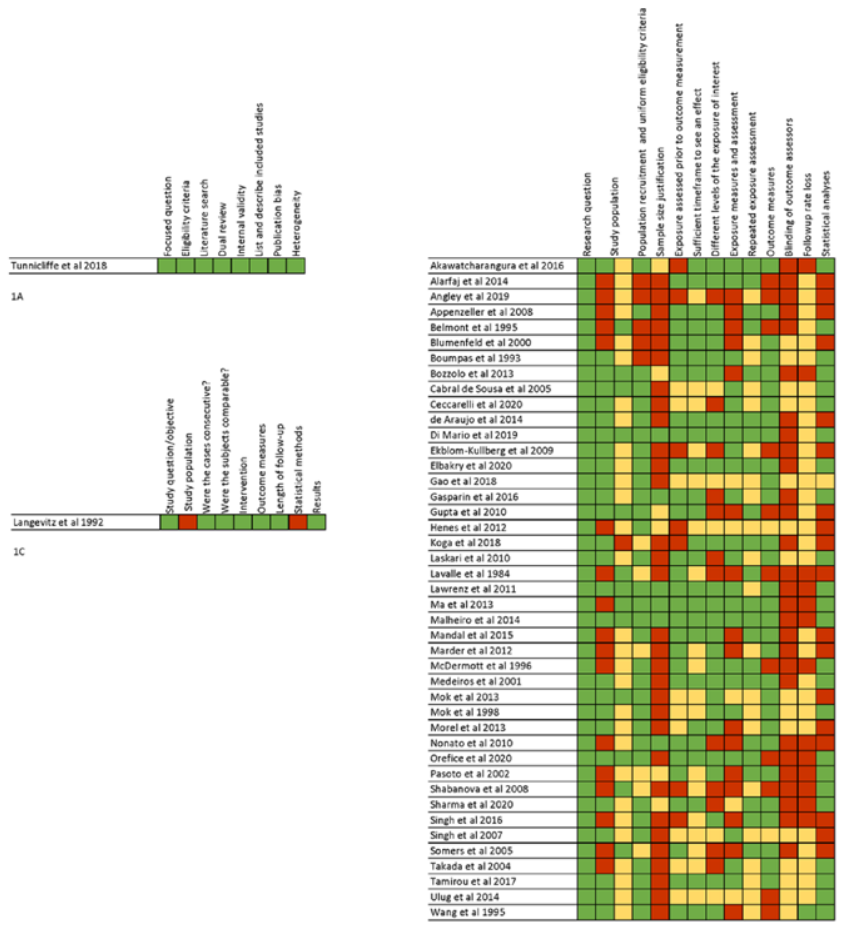

Disclosure of Interests: None declared

DOI: 10.1136/annrheumdis-2021-eular.937

\section{POS0717 AUTOIMMUNE LIVER DISEASE IN ANTICENTROMERE ANTIBODY POSITIVE PRIMARY SJOGREN'S SYNDROME}

B. Chalcev ${ }^{1}$, A. Torgashina ${ }^{1}$, E. Sokol ${ }^{1}$, J. Khvan ${ }^{1}$, V. Vasiliev ${ }^{2}$, S. Palshina ${ }^{3} .{ }^{1}$ V.A. Nasonova Research Institute of Rheumatology, Laboratory of Intensive Care of
Rheumatic Diseases, Moscow, Russian Federation; ${ }^{2}$ MEDSI, Department of Rheumatic Diseases, Moscow, Russian Federation; ${ }^{3}$ Clinic of Heart and Joint Diseases, Department of Rheumatic Diseases, Moscow, Russian Federation

Background: studies have shown that anticentromere antibody (ACA) positivity in primary Sjogren's syndrome (pSS) is associated with autoimmune liver diseases, most often primary biliary cholangitis (PBC) and autoimmune hepatitis $(\mathrm{AlH})[1,2,3]$, but detailed characteristics of the frequency and severity of liver disease in these patients is not presented in the literature.

Objectives: to identify the frequency, structure and characterize the course of autoimmune liver diseases in pSS+ACA.

Methods: we observe 82 patients with pSS+ACA. The diagnosis of pSS was established on the basis of Russian 2001 criteria, SSc was excluded based on the ACR/EULAR 2013 criteria [4]. 18 of 82 patients (22\%) had a persistent increase in alkaline phosphatase, 11 of them were positive for antimitochondrial antibodies (AMA) and, according to the recommendations of the American Association for the Study of Liver Diseases [5], they were diagnosed with PBC. 7 of 18 patients were AMA-negative, 2 of them had a liver biopsy and the diagnosis of AMA-negative PBC was confirmed, 4 patients who did not have a liver biopsy and 1 patient with hepatitis $B$ were excluded from the study. Also, in 6 of 64 patients without signs of liver damage, an increase in AMA was detected, in 1 of them a liver biopsy was performed and the diagnosis of PBC was confirmed. Thus, the group of patients with $\mathrm{pSS}+\mathrm{ACA}$ and autoimmune liver diseases included 19 patients: 12 patients with AMA-positive PBC, 2 patients with AMA-negative PBC and 5 patients with asymptomatic AMA positivity.

Results: The median follow-up for 19 patients with pSS+ACA and autoimmune liver diseases was 4 years. AMA were detected in $89.5 \%$ of patients, an increase in IgM - in 42.1\%, an increase in ALT / AST - 63.2\%, a decrease in albumin, prothrombin index and cytopenia - $15.8 \%$ (were associated with the development of liver cirrhosis). In most cases, the clinical course of liver disease was characterized by an asymptomatic, slowly progressing course, with no signs of progression during observation. Cirrhosis and portal hypertension were detected in $15.8 \%$ of patients, hepatic encephalopathy - in 10.5\%. Liver biopsy was performed in 9 patients, PBC was diagnosed in all cases (overlap syndrome with AlH was established in 3 cases). Assessment of PBC histological stages showed signs of stage 1 in 5 patients, stage 2 in 1 patient, stage 3 in 3 patients. Observation of 5 patients with stage $1 \mathrm{PBC}$ and 5 AMA-positive patients without signs of liver damage (median follow-up was 2 years), showed the absence of clinical, laboratory and instrumental progression of liver disease, which is why we believe that these patients have epithelitis of the biliary ducts as manifestation of glandular lesions in pSS, but not PBC.

Conclusion: autoimmune liver diseases in pSS+ACA are detected in $23.2 \%$ of patients, most of whom develop PBC and epitheliitis of the biliary ducts with the same frequency, less often overlap syndrome of $\mathrm{PBC}$ and $\mathrm{AlH}$, and characterized by a mild, slowly progressing course and rarely lead to liver cirrhosis.

\section{REFERENCES:}

[1] Masako Kita et al. Abnormal Liver Function in Patients with Sjogren's Syndrome. Acta Med. Nagasaki 41: 31-37.

[2] Baldini, Chiara et al. "Overlap of ACA-positive systemic sclerosis and Sjögren's syndrome: a distinct clinical entity with mild organ involvement but at high risk of lymphoma." Clinical and experimental rheumatology vol. 31,2 (2013): 272-80

[3] Bournia, Vasiliki-Kalliopi K et al. "Anticentromere antibody positive Sjögren's Syndrome: a retrospective descriptive analysis." Arthritis research \& therapy vol. 12,2 (2010): R47. doi:10.1186/ar2958.

[4] van den Hoogen, Frank et al. "2013 classification criteria for systemic sclerosis: an American College of Rheumatology/European League against Rheumatism collaborative initiative." Arthritis and rheumatism vol. 65,11 (2013): 2737-47. doi:10.1002/art.38098

[5] Lindor, Keith D et al. "Primary Biliary Cholangitis: 2018 Practice Guidance from the American Association for the Study of Liver Diseases." Hepatology (Baltimore, Md.) vol. 69,1 (2019): 394-419. doi:10.1002/hep.30145.

Disclosure of Interests: None declared

DOI: 10.1136/annrheumdis-2021-eular.949

\section{\begin{tabular}{|l|l}
\hline POS0718 CLINICAL CHARACTERISTICS ASSOCIATED \\
\hline
\end{tabular} WITH GLANDULAR INVOLVEMENT EVALUATED BY SALIVARY GLAND ULTRASONOGRAPHY IN SJÖGREN'S SYNDROME}

T. Shimizu $^{1}$, H. Nakamura ${ }^{1}$, Y. Takagi ${ }^{2}$, S. Y. Nishihata ${ }^{1}$, R. Sumiyoshi ${ }^{1}$, T. Igawa ${ }^{1}$, T. Koga ${ }^{1}$, S. Y. Kawashiri ${ }^{1}$, N. Iwamoto ${ }^{1}$, K. Ichinose ${ }^{1}$, M. Tamai ${ }^{1}$, T. Origuchi ${ }^{1}$, A. Kawakami ${ }^{1}{ }^{1}$ Nagasaki University Graduate School of Biomedical Sciences, Department of Immunology and Rheumatology, Nagasaki, Japan; ${ }^{2}$ Nagasaki University Graduate School of Biomedical Sciences, Department of Radiology and Cancer Biology, Nagasaki, Japan 